This is an author produced version of a paper published in Clinical Physiology and Functional Imaging. This paper has been peer-reviewed but does not include the final publisher proof-corrections or journal pagination.

Citation for the published paper:

Laub, Michael and Midgren, Bengt.

"The effects of nocturnal home mechanical ventilation on daytime blood gas disturbances."

Clinical Physiology and Functional Imaging, 2006, Vol: 26, Issue: 2, pp. 595-606.

http://dx.doi.org/10.1111/j.1475-097X.2006.00648.x

Access to the published version may require journal subscription.

Published with permission from: Blackwell Synergy 


\section{The effects of Nocturnal Home Mechanical Ventilation on Daytime Blood Gas Disturbances}

Michael Laub MD ${ }^{1}$ and Bengt Midgren MD PhD, Ass Prof ${ }^{1,2}$

${ }^{1}$ Dept of Respiratory Medicine, ${ }^{2}$ Lund Sleep Study Group, University Hospital, Lund, Sweden

Correspondence:

Bengt Midgren

Dept of Respiratory Medicine

University Hospital

SE-221 85 Lund

Sweden

e-mail: bengt.midgren@med.lu.se

Short title: HMV and daytime blood gases 


\section{Summary}

Introduction: In chronic alveolar hypoventilation previous studies in selected patient groups have shown that nocturnal home mechanical ventilation (HMV) may result in improved chronic blood gas disturbances during daytime spontaneous breathing. We wished to examine the effects of this treatment in a large non-selected patient material prospectively followed in a national multicentric register.

Material: The material comprised 288 patients from a broad diagnostic spectrum. We looked at the blood gases and vital capacity before the patients electively initiated HMV and at the first register-recorded follow up after 6-24 months.

Results: We found statistically significant improvements in PO2 and PCO2 (approximately $1 \mathrm{kPa}$ in both) and in base excess, but no changes in vital capacity or calculated alveolo-arterial gradient. All changes were independent of the observation period and only weakly diagnose-related.

Discussion: Our findings extend those of previous studies, showing a relatively early and apparently stable improvement in blood gases after starting and continuing nocturnal HMV. The equal extent of the improvements in blood gases among all the diagnostic groups including the neurological patients with progressive diseases was unexpected.

Conclusion: In 288 patients starting nocturnal HMV electively we found significant improvements in daytime blood gases after 6-24 months. There were no changes in vital capacity or calculated alveolo-arterial gradient. Keywords: hypoventilation, long term ventilation, hypercapnia, hypoxemia. 


\section{Introduction}

The last two decades have seen a substantial rise in the number of patients on home mechanical ventilation (HMV) and nowadays this treatment, usually provided as positive pressure non-invasive ventilation (NIV), is well established in patients with chronic alveolar hypoventilation. It is generally assumed that HMV can prolong survival and improve quality of life in many chest wall and neuromuscular diseases (Simonds, 2003). In other situations it can palliate symptoms of nocturnal hypoventilation and breathlessness (Perrin et al., 2004).

Previous studies have convincingly shown that nocturnal NIV results in improved chronic blood gas disturbances during daytime spontaneous breathing (Garay et al., 1981; Elliot et al., 1991; Simonds et al., 1998; Annane et al., 1999; Mellies et al., 2003). However, the patient materials were relatively small (8-30 patients) and did not represent the entire diagnostic spectrum in patients with chronic hypoventilation.

In this study, based on a large patient material from a national register, we wanted to investigate the long-term effects of nocturnal HMV on lung function and blood gases during daytime spontaneous breathing. Particularly we questioned if the impact of the ventilatory support was related to the diagnosis or to the extent of the baseline blood gas abnormality. 


\section{Methods}

Our database was the Swedish Home Mechanical Ventilation Register (Midgren et al., 2000). A closer description of the enlistment of patients in the register is given in reference (Laub et al., 2004). The accumulated number of patients in the register was 1667 as at January 2004 and 1133 individuals were at that moment reported to be alive on a ventilator 6-24 hours a day. The overall treatment prevalence of HMV in Sweden is thus 14/100.000. For patients starting therapy after January $1^{\text {st }} 1996(n=970)$, we collect data on blood gases (spontaneous breathing, room air) and vital capacity at base line and at annual follow ups.

For the purpose of this study, the patients were eligible only if they commenced elective nocturnal ventilatory support after January $1^{\text {st }} 1996$, and were ventilated less than 12 hours per day and had sufficient reported baseline data on blood gases (spontaneous breathing, room air) and lung function ( $\mathrm{N}=741$ patients).

Although most patients were reported to be alive and still on HMV after one year, we have acceptable follow up blood gas and lung function data in 288 patients only, who consequently comprise the patient material.

In the register the patients are divided into 7 main diagnostic groups as follows:

a. Pulmonary disease, b. Neuromuscular disease, c. Post-polio hypoventilation, d. Pickwickian syndrome, e. Tuberculosis sequelae, e. Scoliosis and f. Other 
diseases. The latter group consists of patients with high cervical lesions, central hypoventilation and a medley of other diagnoses.

The time span from embarking HMV to the first register-reported clinical follow up including blood gas sampling and lung function testing is supposed to be 12 months. In practice this time framework is flexible, and the follow up times in this study vary between 6-24 months. In order to detect any time related changes in blood gases, we broke down the follow up data in 3 parts depending on the time span the patients had been on ventilatory support until first follow up. The collection of data at follow up was performed under the same conditions as in the collection of base line data, that is the patients had been breathing room air spontaneously for at least two hours.

Calculation of vital capacity in percent of the predicted value (VC\%pred) was done according to (Berglund et al. 1963). The alveolo-arterial gradient was calculated using the approximate formula: $20-\mathrm{PO} 2-1.25$ x PCO2. We carried out statistical analyses using basic descriptive statistics, tests for normal distribution of the material in each group, which allowed us to make use of t-test to compare the groups separately and analysis of variance to test multiple groups.

\section{Results}


Demographic information of the entire material and the 7 diagnostic groups are displayed in table 1 , which also contains information of the number of hours the patients are ventilated.

The blood gas data and VC\%pred before embarking on ventilatory support and at follow up after mean(SD) 13.2(4.7) months for all patients is given in table 2 . The increase in $\mathrm{PO} 2$ and reduction in $\mathrm{PCO} 2$ and base excess $(\mathrm{BE})$ is statistically significant $(p<0.001$ in all 3 parameters). There was no significant change in vital capacity or alveolo-arterial gradient.

The alterations in PO2 and PCO2 over time in each of the diagnostic groups are shown in figure 1. All changes from start of HMV to follow up are statistically significant with the sole exception of the rise in $\mathrm{PO} 2$ in the scoliosis group. Finally, in figure 2 we display the subdivision of the material according to the time span to the follow up. It is seen that the improvements in blood gases are statistically significant at all time points and independent of the time span studied.

\section{Discussion}

In this study, comprising 288 patients compliant with HMV therapy predominantly yet not exclusively with extrapulmonary respiratory impairment, we found a significant but incomplete normalization in daytime blood gases (spontaneous breathing, room air) after the provision of nocturnal home 
mechanical ventilation for 6-24 months. There was no concomitant change in vital capacity or calculated alveolo-arterial gradient.

A national register without exclusion criteria has drawbacks and advantages when compared to a formal clinical trial. Patients are not following a specific protocol and differences in treatment criteria may be due to bias in patient selection or to local procedures in treatment and follow-up. A register-based study may be severely biased in case of under-reporting.

The main weakness of our study is that it is based on a register with many incomplete follow up data. In an attempt to explain the large number of missing data, we enquired some of the HMV-centres directly. The commonest reported reason accounting for missing data was that patients cancelled or postponed the regular examinations or, the other way round, the centres did so because of shortage of capacity. Furthermore, we found that some patients at the follow up time had started continuous oxygen therapy or had increased the number of hours on ventilatory support, two facts that rule out the option to compare blood gases on spontaneous ventilation in room air. Mortality can account for less than $10 \%$ of the missing data and, finally, a small proportion of the patients had moved and were lost for follow up. In the missing patients we compared the base line data with the corresponding data in the included patients and found no difference with respect to age and diagnostic distribution, but adequately followed up patients presented slightly worse blood gases at therapy start. 
Nevertheless, we believe that our results are valid and they are well in line with previous observations in smaller patient materials. The strength of our study is that it represents a broad diagnostic spectrum collected from many centres.

The blood gas pattern in our patients is that of chronic hypoventilation. The changes towards normalization after 6-24 months were not accompanied by any improvement in vital capacity. Since our patients did not undergo any extended studies on ventilatory muscle strength or endurance, we can only speculate about the mechanisms behind the improvements in blood gases. Restoration of hypercapnic ventilatory response seems to be the most plausible explanation (Annane et al., 1999; Dellborg et al., 2000).

Another interesting observation is that there was no relationship between baseline blood gas abnormality and the extent of the normalization. We aimed at including only stable patients, where HMV was started electively. A large number of misclassified patients with acute rather than chronic hypoventilation would have resulted in a greater degree of reduction in PCO2 in the most hypercapnic patients. Since this was not the case, we believe that our assumption of clinical stability is valid. The level of the base excess also speaks in favour of this assumption.

As demonstrated in figure 2 the improvements in blood gases seem to appear relatively early and remain at the same level for 16-24 months. Still we have only shown this indirectly, as we only have data from one follow up in each 
patient. A long-term stability has, however, been shown for patients with stable thoracic deformities (Duiverman et al., in press).

Interestingly, though the improvement in PO2 in the scoliosis patients did not reach statistical significance, there was no evident difference between the diagnostic

groups with respect to the blood gas improvement. This is somewhat unexpected since some of the neurological diseases are progressive, and this group, therefore, could be expected to be more therapy resistant. It must be emphasized that our data are valid only for those patients who could be maintained on HMV for 12 hours per day or less.

We conclude that our patients with chronic hypoventilation representing a broad spectrum of underlying diseases improved their daytime PO2 and PCO2 with approximately $1 \mathrm{kPa}$ without a concomitant improvement in vital capacity after 6-24 months on nocturnal home mechanical ventilation. 


\section{References}

Annane D, Quera-Salva MA, Lofaso F et al. Mechanisms underlying effects of nocturnal ventilation on daytime blood gases in neuromuscular patients. Eur Respir (1999); 13: 157-162.

Berglund E, Birath G, Bjure J et al. Spirometric studies in normal subjects. Forced expirograms in subjects between 7 and 70 years of age. Acta Med Scand (1963); 173: 185-92.

Elliot MW, Mulvey DA, Moxham J et al. Domiciliary nocturnal nasal intermittent positive pressure ventilation in COPD: mechanisms underlying changes in arterial blood gas tensions. Eur Respir J (1991); 4: 1044-1052.

Dellborg C, Olofson J, Hamnegaard $\mathrm{CH}$ et al. Ventilatory response to $\mathrm{CO} 2$ rebreathing before and after nocturnal nasal intermittent positive pressure ventilation in patients with chronic alveolar hypoventilation. Resp Med (2000); 94: 1154-1160.

Duiverman ML, Bladder G, Meinesz AF et al. Home mechanical ventilatory support in patients with restrictive ventilatory disorders: a 48-year experience. Accepted for publication in Respiratory Medicine.

Garay SM, Turino GM and Goldring RM. Sustained reversal of chronic hypercapnia in patients with alveolar hypoventilation syndromes: long-term maintenance with noninvasive nocturnal ventilation. Am J Med (1981); 70: 269-274. 
Laub M, Berg S, Midgren B. Home mechanical ventilation in Sweden - inequalities within a homogenous health care system. Resp Med (2004); 98: $38-42$.

Mellies U, Ragette R, Schwake CD et al. Long-term noninvasive ventilation in children and adolescents with neuromuscular disorders. Eur Respir J (2003); 22: $631-636$.

Midgren B, Olofson J, Harlid R, et al. Home mechanical ventilation in Sweden, with reference to Danish experiences. Resp Med (2000); 94: 135-138.

Perrin C, Unterborn JN, D'Ambrosio C et al. Pulmonary complications of chronic neuromuscular diseases and their management. Muscle Nerve (2004); 29: 5-27.

Simonds AK, Muntoni F, Heather S et al. Impact of ventilation on survival in hypercapnic Duchenne muscular dystrophy. Thorax (1998); 53: 949-952. Simonds AK. Home ventilation. Eur Respir J (2003); 22: Suppl. 47, 38s-46s. 


\section{Table 1}

\begin{tabular}{c|cccccccc}
\hline Patient group & All & Pulm & Neuro & Polio & Pickw & Scol & TBC & Other \\
\hline N & 288 & 20 & 58 & 36 & 76 & 41 & 31 & 26 \\
Age & $59(15)$ & $62(8)$ & $47(18)$ & $67(8)$ & $59(12)$ & $59(14)$ & $72(5)$ & $60(15)$ \\
HMV 7-8h/day & $90 \%$ & $95 \%$ & $90 \%$ & $89 \%$ & $99 \%$ & $76 \%$ & $81 \%$ & $92 \%$ \\
HMV 8-12h/day & $10 \%$ & $5 \%$ & $10 \%$ & $11 \%$ & $1 \%$ & $24 \%$ & $19 \%$ & $8 \%$ \\
\hline
\end{tabular}

Legend to Table 1.

Demographic information. Pulm: Pulmonary diseases, Neuro: Neuromuscular diseases, Polio: Post-polio hypoventilation, Pickw: Pickwickian syndrome, Scol:

Scoliosis, TBC: Tuberculosis. HMV: Home mechanical ventilation. Age is expressed as mean(SD). 


\section{Table 2}

\begin{tabular}{l|ll}
\hline & Baseline & Follow up \\
\hline PO2 kPa & $8.0(1.7)$ & $9.1(1.7)^{* * *}$ \\
PCO2 kPa & $7.2(1.2)$ & $6.3(0.9)^{* * *}$ \\
BE mmol x I-1 & $6.5(3.2)$ & $4.2(3.0)^{* * *}$ \\
VC\%pred & $43(17)$ & $45(18) \mathrm{NS}$ \\
A-a gradient kPa & $3.0(1.7)$ & $3.0(1.7) \mathrm{NS}$ \\
\hline
\end{tabular}

Legend to Table 2.

Alterations in blood gases and vital capacity in percent of the predicted value after 13.2(4.7) months in 288 HMV patients. BE: Base excess, VC\%pred: Vital capacity in percent of the predicted value, A-a gradient: Alveolo-arterial gradient.

Figures are expressed as mean(SD).

Three asterisks state $p<0.001$, NS: Statistically non significant. 
HMV and Daytime Blood Gas

Figure 1

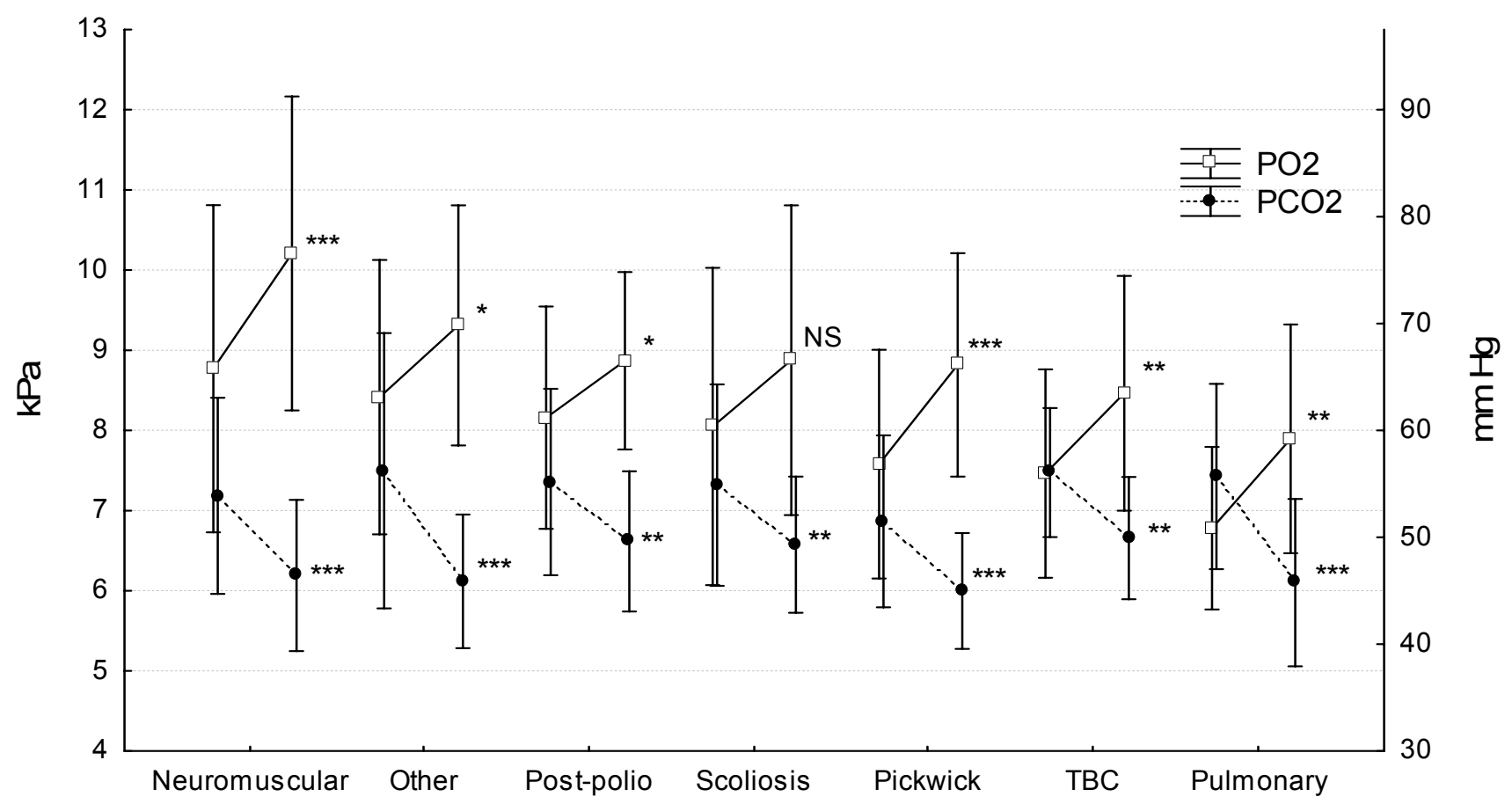


HMV and Daytime Blood Gas

Figure 2

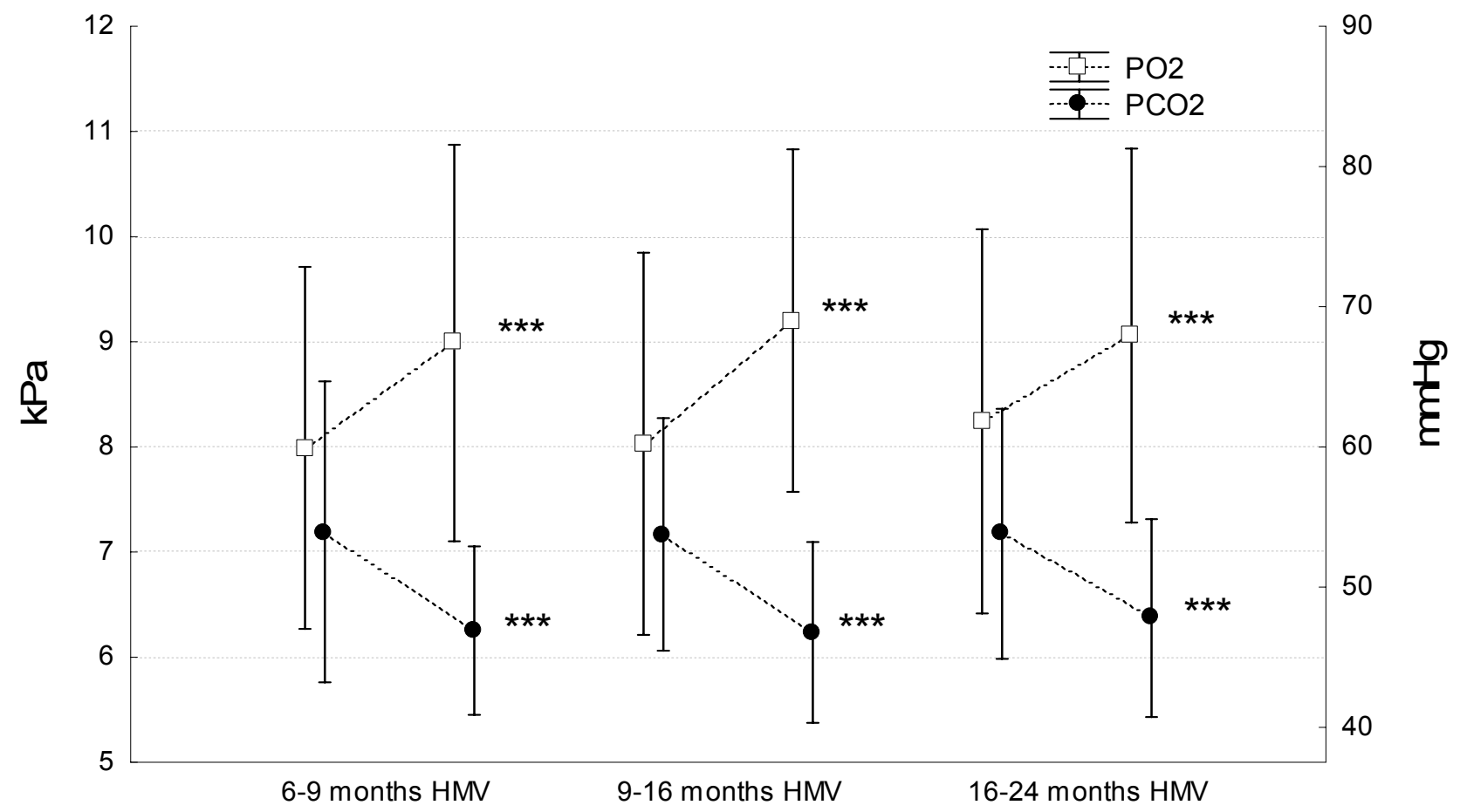


Legend to Figure 1.

Alterations in PO2 and PCO2 after initiation of HMV in 288 patients divided in 7 diagnostic groups ranked according to the baseline PO2. The mean(SD) observation period for all patients was 13.2(4.7) months. The number of asterisks states the level of statistical significance: $1: p<0.05,2: p<0.01,3: p<0.001$. NS: Statistically non significant.

\section{Legend to Figure 2.}

Alterations in PO2 and PCO2 after initiation of HMV in 288 patients displayed in 3 groups depending on the duration of the ventilatory support. The number of asterisks states the level of statistical significance (as in figure 1). 